\title{
Highly Birefringent Optical-Fiberized Slot Waveguide for Miniature Polarimetric Interference Sensors: A Proposal
}

\author{
Jun-long Kou, Wei Guo, Fei Xu, and Yan-qing Lu, Senior Member, IEEE
}

\begin{abstract}
A new scheme of highly birefringent fiberized slot waveguide (FSW) by post-processing a non-birefringent circular microfiber is proposed for polarimetric interference sensors with ultrasmall sensor heads (tens of micrometers). The different sensing properties of an FSW polarimetric interferometer based on a typical fiber loop mirror are investigated and discussed in detail for refractive index, temperature, and strain measurement. The sensitivity can achieve a high level of $>5 \times 10^{4} \mathrm{~nm} /$ refractive index unit (RIU), $>5 \mathrm{~nm} /{ }^{\circ} \mathrm{C}$, and $\sim 1 \mathrm{pm} / \mu \varepsilon$ for refractive index, temperature, and strain sensing, respectively. Moreover, its strain sensitivity has a large tuning range from negative to positive, and can be used as an ultracompact and highly efficient strain compensator. Together with its high birefringence, unique geometry, and extremely small size, FSW presents wide potential applications in physical, biological and/or chemical sensing areas.
\end{abstract}

Index Terms-High birefringence, sensor, slot waveguide.

\section{INTRODUCTION}

$\mathbf{O}$ PTICAL fiber technology is one of the most effective methods for sensing. Various kinds of sensors utilizing optical fibers have been investigated, such as Bragg gratings, Mach-Zehnder and polarimetric interference that are based on highly birefringent (Hi-Bi) fibers. The Hi-Bi fibers are polarization maintaining fibers where the linear polarization states are structurally maintained. They are easy to be applied in optical sensing, by implementing a polarimetric interference such as a fiber loop mirror or Sagnac loop [1], [2]. Typical Hi-Bi fibers such as Bow-Tie [3], PANDA [4], and Hi-Bi photonic crystal fibers [5], have been widely studied and applied in strain or temperature measurement and other sensors. However, these Hi-Bi fibers with their diameters of hundreds of micrometers seriously limit the sensors' size and are difficult to be used in refractometers as biological and/or

Manuscript received July 7, 2011; revised November 10, 2011; accepted November 15, 2011. Date of publication November 29, 2011; date of current version April 20, 2012. This work was supported in part by the National 973 Program under Contract 2010CB327803, Contract 2011CBA00200, and Contract 2012CB921803, the Natural Science Foundation of China under Program 11074117 and Program 60977039, and the Fundamental Research Funds for the Central Universities. The Associate Editor coordinating the review of this paper and approving it for publication was Dr. M. Nurul Abedin.

The authors are with the Department of Materials Science and Engineering, College of Engineering and Applied Sciences and National Laboratory of Solid State Microstructures, Nanjing University, Nanjing 210093, China (e-mail: feixu@nju.edu.cn; yqlu@nju.edu.cn).

Color versions of one or more of the figures in this paper are available online at http://ieeexplore.ieee.org.

Digital Object Identifier 10.1109/JSEN.2011.2177521 chemical sensors because of weak available evanescent field outside, even for the D-type Hi-Bi fiber [6]. Subwavelength $\mathrm{Hi}-\mathrm{Bi}$ microfibers are promising elements to meet the increasing demands of nano-photonic sensors and to decrease the sensors' size while keeping large evanescent field. With the fast development of microfiber fabrication technology, several approaches have been reported to fabricate Hi-Bi microfibers: such as adiabatically tapering conventional polarization maintaining (PM) fibers [7] and rectangular-shaped fibers [8], [9]. However, it presents great challenges to keep these asymmetric geometries during tapering which is a serious concern.

Recently, a planar Hi-Bi slot-waveguide that consists of a thin, low-index "slot" fabricated in a high-index waveguide has attracted great attention [10]. This opens up the interior region of the waveguide, where the electric field intensity is the highest, perfect for sensing [11]. Combining current mature technology of drawing circular microfibers (CMFs) and micromachining, it is possibly easier to realize a $\mathrm{Hi}-\mathrm{Bi}$ fiberized slot waveguide (FSW) by post-processing a non-birefringent CMF. With the unique hybrid structure, FSWs will show great potentials for miniature fiberized sensors, accompanied by a lot of advantages such as high birefringence, large evanescent field, super-compact size, flexible design, natural input/output ends with standard fibers, and simplified fabrication.

In this paper, we propose a possible approach to realize a Hi-Bi microfiber with a slot inside by post-processing a non-birefringent CMF and investigate a miniature FSW-based polarimetric interference sensor with the ultra-small sensor head for refractive index, temperature, and strain measurement. Its size can be minimized to tens of micrometers, much smaller than current polarimetric interference sensors. Taking benefit of the special and flexible structure, its sensitivity for liquid refractive index can reach an extremely high level of $10^{4} \mathrm{~nm} / \mathrm{RIU}$ (refractive index unit), its liquid temperature sensitivity $\left(>5 \mathrm{~nm} /{ }^{\circ} \mathrm{C}\right)$ can be much better than recent results in fiber temperature sensors, and its strain sensitivity $(\sim 1 \mathrm{pm} / \mu \varepsilon)$ can be comparable with or better than recent results utilizing the same sensing mechanism. It is worth remarking that the strain sensitivity also has a large tuning range from negative to positive, which means that it can be used as an ultra-compact and highly efficient strain compensator in other fiber systems. The FSW-based sensor may find more potential in physical, biological and/or chemical applications with its unique geometry and extremely small size. 


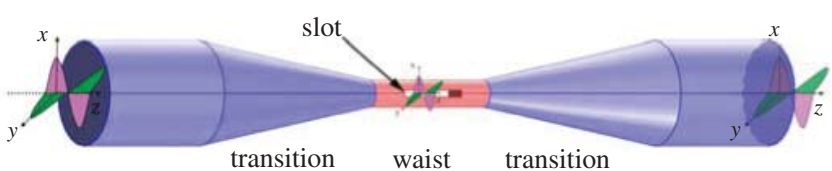

(a)

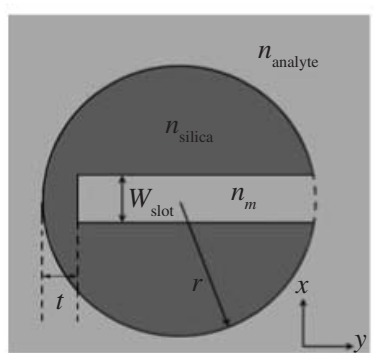

(b)

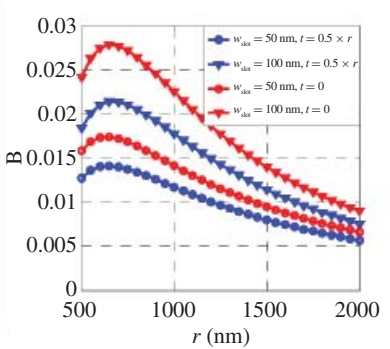

(c)
Fig. 1. (a) Schematic of the FSW. (b) Cross section of the FSW with $r$ the radius of the microfiber, other parameters are presented in the picture. (c) Birefringence of the FSW as a function of its geometry parameters.

\section{HI-BI FSWS AND FSW-BASED POLARIMETRIC INTERFERENCE SENSORS}

Figure 1(a) shows the schematic of the Hi-Bi FSW. It is a conventional microfiber with a slot located at the waist. Here, the indices of the microfiber, medium in the slot and outside environment are denoted by $n_{\text {silica }}, n_{m}$, and $n_{\text {analyte }}$ respectively. Other parameters used in this paper are presented in the cross section diagram (Fig. 1(b)). All the calculation work is done at the wavelength of $1550 \mathrm{~nm}$ by using a fullvectorial finite-element analysis.

Due to the broken of the circular symmetry observed in fiberized slot waveguides, high birefringence $(B)$ above $10^{-2}$ can be achieved in FSWs as shown in Fig. 1(c). As the microfiber radius increases, birefringence first becomes larger and then decreases. Our calculation results show that it can even reach a very high level of $4 \times 10^{-2}$ at $r=1 \mu \mathrm{m}$, $W_{\text {slot }}=400 \mathrm{~nm}$, and $t=0$.

Throughout this paper, we investigate the sensing properties of the FSWs by employing the typical polarimetric interference setup, a fiber loop mirror as shown in Fig. 2 [12]. The transmission of the light with respect to the operation wavelength $(\lambda)$ can be obtained [12]:

$$
T(\lambda) \propto\left[\cos \left(\frac{\pi B L}{\lambda}\right)\right]^{2}
$$

where $L$ is length of the FSW. Due to the high birefringence of the FSW ( $\sim 10^{-2}$ shown in Fig. 1(c)), the length of the slot up to several hundreds of micrometers is enough for sensing according to Eq. 1. Because at this length, a reasonable free spectral range can be achieved, considering the common light sources found in labs. Such a structure can be obtained using modern micro-fabrication method, such as focused ion beam (FIB) or deep-UV lithography [13]. Due to the ultra-small volume of the sensing area, it is important to highlight that these FSWs may be excellent candidate for physical, biological and/or chemical sensing.

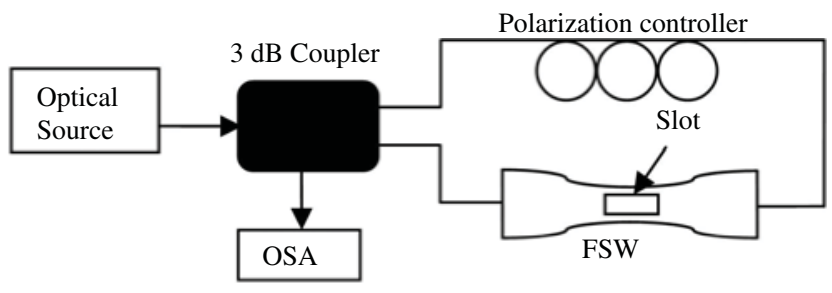

Fig. 2. Schematic of a typical Hi-Bi fiber loop mirror for sensing based on polarimetric interference.

\section{FSWS: ENHANCED SENSITIVITY OF REFRACTIVE INDEX}

Previously, Hi-Bi fibers (with diameters $\sim 125 \mu \mathrm{m}$ as mentioned above) can hardly be used as elements in refractive index sensors because that the light field has no access to the sensing medium. Here, we investigate the sensing property of the FSW which provides a natural channel for liquid/gas refractive index measurement. The sensing mechanism employs its relatively high birefringence (on the order of $10^{-2}$ ) as well as its large evanescent field both outside the silica fiber and inside the slot. In all these interference-based sensors, sensitivity of the analyte ( $\left.S_{\text {analyte }}\right)$ is defined as the shift of the interference wavelength $\lambda_{0}$ with respect to the analyte (assumed to be water, $n_{\text {analyte }}=n_{\text {water }}$ ) index change:

$$
S_{\text {analyte }}=\frac{\partial \lambda_{0}}{\partial n_{\text {analyte }}}=\frac{\partial \lambda_{0}}{\partial B} \frac{\partial B}{\partial n_{\text {analyte }}}=\frac{\lambda_{0}}{B} \frac{\partial B}{\partial n_{\text {analyte }}} .
$$

Figure 3(a) and (b) show the sensitivity of the FSWs as a function of the microfiber radius. As can be deduced, sensitivity as high as $10^{3}$ to $10^{4} \mathrm{~nm} / \mathrm{RIU}$ is achieved with microfibers of different radius which can be easily obtained using the mature fiber tapering technique. From these figures, we can also see that a bigger slot width and height (that is smaller $t$ ) results in a higher sensitivity. However, according to our calculation, for $W_{\text {slot }}$ larger than $100 \mathrm{~nm}$, the microfiber can hardly support the propagating wave and causes intolerable loss when immersed in water. What is more, by comparing the two green lines in Fig. 3 (a) and (b), $t$ has a small effect on sensitivity when it is less than $0.5 \times r$. As $t$ increases, both sensitivity and birefringence become smaller. Sensitivity changes quickly with microfiber radius, thus controlling the radius of the microfiber to sub-wavelength level is a key point in achieving a high sensitivity.

Microfiber refractive index sensors based on fiber Bragg gratings with the same radius have a sensitivity less than $10^{3} \mathrm{~nm} / \mathrm{RIU}$ as pointed out in Ref. [14]. Here, attributed to the unique structure, we can obtain an extremely high sensitivity $\left(>10^{4} \mathrm{~nm} / \mathrm{RIU}\right)$ in a wide range of geometry dimensions based on the high birefringence and large evanescent field of the FSWs.

\section{FSWS: ENHANCED SENSITIVITy OF TEMPERATURE}

Various types of fiber temperature sensors have been developed because of its light weight, immunity to electromagnetic interferences and flexibility of directly embedding into the system. Among them, the one based on long period gratings is attractive due to its high sensitivity; however, its size is 


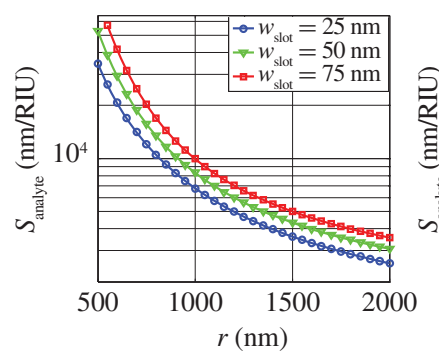

(a)

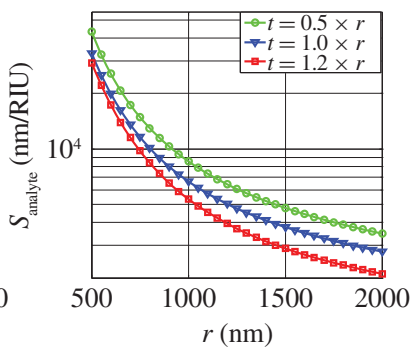

(b)
Fig. 3. Sensitivity of the FSWs as a function of microfiber radius (a) at different slot width $\left(W_{\text {slot }}\right)$ with $t=0$ and (b) at different height $(2 r-t)$ of the slot with $W_{\text {slot }}=50 \mathrm{~nm}$.

too long and it easily suffers from the bending effect [15]. The use of fiber Bragg gratings has provided us with sensors possessing wavelength multiplexing feature; but the temperature sensitivity $\left(S_{T}\right)$ is greatly limited $\left(\sim 10 \mathrm{pm} /{ }^{\circ} \mathrm{C}\right)$ [16]. The temperature sensor using a $\mathrm{Hi}$-Bi polarimetric interferometer has attracted more and more attention owing to its stability, flexibility, and very high sensitivity $\left(\sim 1 \mathrm{~nm} /{ }^{\circ} \mathrm{C}\right)$ [2], [17]. Of course, the sizes of these temperature sensors are still limited by the conventional thick Hi-Bi fibers.

Here, we investigate the temperature sensitivity $\left(\right.$ at $20^{\circ} \mathrm{C}$ ) of the proposed FSW-based polarimetric interferometer. By monitoring the shift of the interference wavelength with respect to the change of the temperature, we define the temperature sensitivity as:

$$
\begin{aligned}
S_{T}= & \frac{\partial \lambda_{0}}{\partial T}=\frac{\partial \lambda_{0}}{\partial B} \frac{\partial B}{\partial T}+\frac{\partial \lambda_{0}}{\partial L} \frac{\partial L}{\partial T} \\
= & \frac{\partial \lambda_{0}}{\partial B}\left(\frac{\partial B}{\partial r} r \frac{1}{r} \frac{\partial r}{\partial T}+\frac{\partial B}{\partial n_{\text {silica }}} \frac{\partial n_{\text {silica }}}{\partial T}+\frac{\partial B}{\partial n_{\text {water }}} \frac{\partial n_{\text {water }}}{\partial T}\right) \\
& +\frac{\partial \lambda_{0}}{\partial L} \frac{\partial L}{\partial T} \\
= & \frac{\lambda_{0}}{B}\left(\frac{\partial B}{\partial r} r \alpha_{T}+\frac{\partial B}{\partial n_{\text {silica }}} \sigma_{T, \text { silica }}+\frac{\partial B}{\partial n_{\text {water }}} \sigma_{T, \text { water }}\right) \\
& +\lambda_{0} \alpha_{T}
\end{aligned}
$$

where $\alpha_{T}\left(5.5 \times 10^{\left.-7 /{ }^{\circ} \mathrm{C}\right)}\right.$ is the thermal expansion coefficient of silica and $\sigma_{T, \text { silica }}\left(1.1 \times 10^{-5} /{ }^{\circ} \mathrm{C}\right), \sigma_{T, \text { water }}\left(-8 \times 10^{-5} /{ }^{\circ} \mathrm{C}\right)$ are the thermo-optics coefficient of the fused silica and water, respectively. According to our calculations shown in Fig. 4(a), thermal expansion effect (the first and fourth part of Eq. 3) contributes little to the total sensitivity $(<2 \%)$, mainly due to the low thermal expansion coefficient of silica. And sensitivity resulting from the refractive index change of water (red line in Fig. 4(a)) is more than five times bigger than that of silica (green line in Fig. 4(a)). In Fig. 4(b) we plot the total temperature sensitivity resulting from both the thermo-optics effect and thermal expansion effect under three different values of $W_{\text {slot }}$. The sensitivity can achieve as high as $5 \mathrm{~nm} /{ }^{\circ} \mathrm{C}$. It is much better than recent results in fiber temperature sensors [2], [17]. It is important to emphasize that the temperature sensitivity has a large dependence on microfiber radius. When $r$ is larger than $1 \mu \mathrm{m}, S_{T}$ becomes lower than $1 \mathrm{~nm} /{ }^{\circ} \mathrm{C}$ and is less dependent on $W_{\text {slot }}$. This is because that as $r$ is comparable to or larger than the wavelength; the light field will be well confined in the silica and slot region.

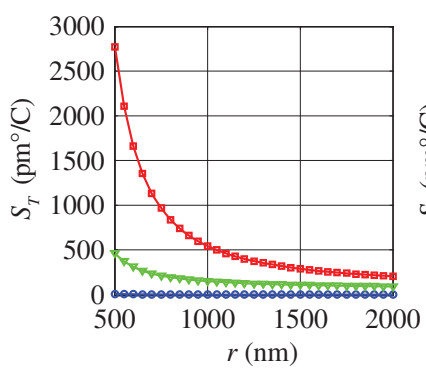

(a)

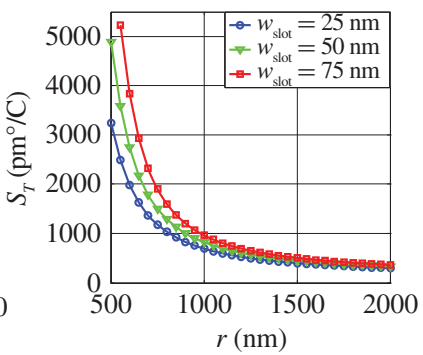

(b)
Fig. 4. (a) Temperature sensitivity at $W_{\text {slot }}=25 \mathrm{~nm}$ and $t=0$, the red, green, and blue lines represent the temperature sensitivity results from refractive index change of water, silica, and structure change of the microfiber and (b) temperature sensitivity at different $W_{\text {slot }}$ with $t=0$, the blue line is the summation of the values of the three lines in (a).

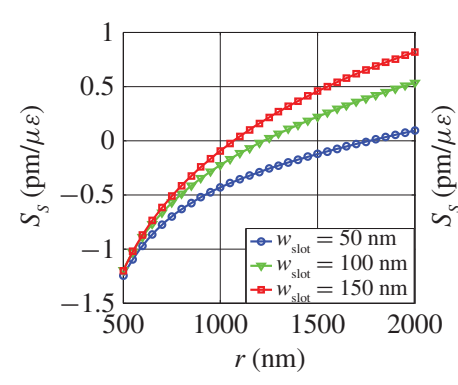

(a)

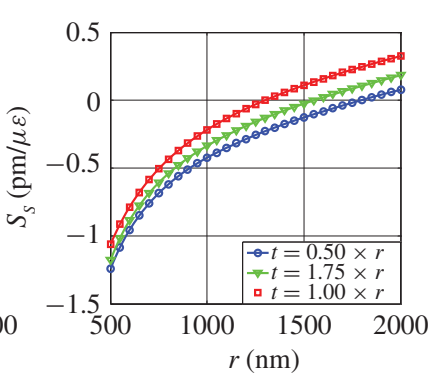

(b)
Fig. 5. Strain sensitivity (a) at $t=0$ under different values of $W_{\text {slot }}$ and (b) at $W_{\text {slot }}=50 \mathrm{~nm}$ under different values of $t$.

Thus the thermo-optics coefficient of silica will play a more important role in affecting $S_{T}$, leading to a lower temperature sensitivity.

\section{FSWS: ENHANCED SENSITIVITy OF STRAIN WITH A LARGE TUNING RANGE}

Conventional Hi-Bi fibers have also been intensively used in strain sensors [18]. Based on mechanics of materials, when longitudinal strain is applied to the FSW, wavelength shift can be estimated as follows:

$$
\begin{aligned}
\Delta \lambda & =\frac{\Delta B}{B} \lambda_{0}+\frac{\Delta L}{L} \lambda_{0}=\lambda_{0}\left(\frac{\partial B}{\partial n_{\text {silica }}} \frac{1}{B} \Delta n_{\text {silica }}+\varepsilon\right) \\
& =\lambda_{0} \varepsilon\left\{\frac{1}{B} \frac{\partial B}{\partial n_{\text {silica }}}\left[-\frac{1}{2} n_{\text {silica }}^{3}\left(p_{12}-v\left(p_{11}+p_{12}\right)\right)\right]+1\right\}
\end{aligned}
$$

The influence due to a change in the fiber diameter (waveguide geometry effect) is small and can therefore be neglected [19]. As can be seen, wavelength shift is proportional to the strain applied to the FSW at a fixed cross section of the fiberized slot waveguide (i.e., at a certain value of $r, t$ and $\left.W_{\text {slot }}\right)$. And strain sensitivity $\left(S_{S}=\Delta \lambda / \varepsilon\right)$ can thus be easily deduced just like what we have discussed in the previous section. Three parameters appearing in Eq. 4, i.e., Poisson's ratio $(v=0.16)$, strain-optics coefficient of silica ( $\left.p_{11}=0.11, p_{12}=0.25\right)$ can be found in Ref. [19]. From Fig. 5, the attainable highest strain sensitivity is about $1 \mathrm{pm} / \mu \varepsilon$, comparable to previous results utilizing the same sensing mechanism [18]. $W_{\text {slot }}$ has a bigger influence on $S_{s}$ 
as $r$ increases. It is worth noting that the strain sensitivity can be both negative and positive by varying the radius, which means that FSW has great potential in an ultra-compact and highly efficient strain compensator for various fiber systems.

\section{DISCUSSIONS}

Slot waveguide based on a planar substrate has been extensively investigated both theoretically and experimentally. Single-slot, double-slot, and multi-slot waveguides have been fabricated and applied in sensing and communication. FSW or slot waveguide based on a CMF is the first time to be presented and discussed in this paper. It can be made by utilizing current advanced micro-machining techniques, particularly FIB micromachining. So far, a number of engineered fiber micro/nano devices by FIB drilling have been fabricated and demonstrated successfully, such as a 3.7- $\mu \mathrm{m}$ fiber-top cantilever which is nearly free standing in air [20], [21], a Fabry-Perot cavity based on a microfiber with a large slot (2- $4 \mu \mathrm{m}$ long and high, located at the position with the local radius $r=2-4 \mu \mathrm{m}$ ) [13], [22], an ultra-short microfiber grating with deep periodic grooves (11 grooves in a microfiber $5 \mu \mathrm{m}$ in diameter, every groove is $1.6 \mu \mathrm{m}$ deep) [23], [24]. These devices have been demonstrated as various sensors such as temperature, refractive index, vibration and displacement sensors, and they are stable, portable, and reliable. Considering these successful examples of FIB-machined microfiber sensors with more complicated and challenging geometries, FSW with a slot tens of nanometers in width can also be fabricated using the similar process and employed as sensors.

Our simulation shows that the length of the slot up to $10 \mu \mathrm{m}$ is enough for sensing according to Eq. 1. Such a short length also makes the fabrication and handling easier. Moreover, the fiber is still mechanically strong with a slot in a diameter of $\sim 1 \mu \mathrm{m}$ provided that $t$ does not approach zero. It is possible but more difficult to fabricate an FSW under much smaller diameter $(<1 \mu \mathrm{m})$. Hoverer, FSW with several micrometer diameters is much easier to be realized and used in sensing. For a $2-\mu \mathrm{m}$-diameter FSW, its birefringence is still very large (0.01-0.02) which is enough for sensing applications. In piratical situations, FSWs $(1-2 \mu \mathrm{m}$ in diameter) with slot (10-20 $\mu \mathrm{m}$ in length) are preferred and suitable to serve as a miniature sensor head. Longer and thinner FSW is not recommended.

By employing the fiber loop mirror setup, FSW based sensor head can be used to measure various physical or chemical parameters with high sensitivity. From our previous work and experience on tapered microfiber sensors with large open-grooves, however, refractive index and temperature measurements should be implemented more easily than strain measurement. Furthermore, if incorporated with fiber Bragg gratings in the microfiber, FSW may open up new possibilities for multi-parameter sensing applications.

\section{CONCLUSION}

We have analyzed in detail the enhanced sensing properties of the proposed FSW utilizing its high birefringence and large evanescent field. It shows a high sensitivity in refractive index, temperature, and strain measurement. Its unique geometry enables the sensor to be tens of micrometers in size, much smaller than other Hi-Bi fiber sensors. The FSW has great potentials in ultra-compact and highly efficient strain compensators because of its special property of the strain sensitivity. The large evanescent field, flexible design, and the slots for liquid/gas flow makes FSW a promising element in future biological and/or chemical areas, nano-photonics and microsystems.

\section{REFERENCES}

[1] O. Frazao, J. M. T. Baptista, and J. L. Santos, "Recent advances in high-birefringence fiber loop mirror sensors," Sensors, vol. 7, no. 11, pp. 2970-2983, 2007.

[2] E. De la Rosa, L. A. Zenteno, A. N. Starodumov, and D. Monzon, "All-fiber absolute temperature sensor using an unbalanced highbirefringence Sagnac loop," Opt. Lett., vol. 22, no. 7, pp. 481-483, 1997.

[3] M. P. Varnham, D. N. Payne, R. D. Birch, and E. J. Tarbox, "Singlepolarization operation of highly birefringent bow-tie optical fibers," Electron. Lett., vol. 19, no. 7, pp. 246-247, Mar. 1983.

[4] T. Hosaka, K. Okamoto, T. Miya, Y. Sasaki, and T. Edahiro, "Lowloss single polarization fibers with asymmetrical strain birefringence," Electron. Lett., vol. 17, no. 15, pp. 530-531, Jul. 1981.

[5] M. Delgado-Pinar, A. Diez, S. Torres-Peiro, M. V. Andres, T. PinheiroOrtega, and E. Silvestre, "Waveguiding properties of a photonic crystal fiber with a solid core surrounded by four large air holes," Opt. Exp., vol. 17, no. 9, pp. 6931-6938, 2009.

[6] O. Frazao, B. V. Marques, P. Jorge, J. M. Baptista, and J. L. Santos, "High birefringence D-type fibre loop mirror used as refractometer," Sens. Actuat. B, vol. 135, no. 1, pp. 108-111, Dec. 2008.

[7] Y. Jung, G. Brambilla, and D. J. Richardson, "Polarization-maintaining optical microfiber," Opt. Lett., vol. 35, no. 12, pp. 2034-2036, 2010.

[8] H. Xuan, J. Ju, and W. Jin, "Highly birefringent optical microfibers," Opt. Exp., vol. 18, no. 4, pp. 3828-3839, 2010.

[9] Y. Jung, G. Brambilla, K. Oh, and D. J. Richardson, "Highly birefringent silica microfiber," Opt. Lett., vol. 35, no. 3, pp. 378-380, 2010.

[10] V. R. Almeida, Q. Xu, C. A. Barrios, and M. Lipson, "Guiding and confining light in void nanostructure," Opt. Lett., vol. 29, no. 11, pp. 1209-1211, 2004

[11] C. A. Barrios, K. B. Gylfason, B. Sanchez, A. Griol, H. Sohlstrom, M. Holgado, and R. Casquel, "Slot-waveguide biochemical sensor," Opt. Lett., vol. 32, no. 21, pp. 3080-3082, 2007.

[12] D. B. Mortimore, "Fiber loop reflectors," IEEE J. Lightw. Technol., vol. 6, no. 7, pp. 1217-1224, Jul. 1988.

[13] J.-L. Kou, J. Feng, L. Ye, F. Xu, and Y.-Q. Lu, "Miniaturized fiber taper reflective interferometer for high temperature measurement," Opt. Exp., vol. 18, no. 3, pp. 14245-14250, 2010.

[14] J.-L. Kou, Z.-D. Huang, G. Zhu, F. Xu, and Y.-Q. Lu, "Wave guiding properties and sensitivity of D-shaped optical fiber microwire devices," Appl. Phys. B, vol. 102, no. 3, pp. 615-619, 2010.

[15] V. Bhatia and A. M. Vengsarkar, "Optical fiber long-period grating sensors," Opt. Lett., vol. 21, no. 9, pp. 692-694, 1996.

[16] Y. Li, M. Yang, D. N. Wang, J. Lu, T. Sun, and K. T. Grattan, "Fiber Bragg gratings with enhanced thermal stability by residual stress relaxation," Opt. Exp., vol. 17, no. 22, pp. 19785-19790, 2009.

[17] J. Yongxing, C. C. Chan, Y. Zhang, X. Dong, and P. Zu, "Temperature sensor based on a pressure-induced birefringent single-mode fiber loop mirror," Meas. Sci. Technol., vol. 21, no. 6, pp. 65204-1-65204-4, 2010.

[18] X. Y. Dong, H. Y. Tam, and P. Shum, "Temperature-insensitive strain sensor with polarization-maintaining photonic crystal fiber based Sagnac interferometer," Appl. Phys. Lett., vol. 90, no. 15, pp. 151113-1-1511133, 2007.

[19] A. Bertholds and R. Dandliker, "Determination of the individual strainoptic coefficients in single-mode optical fibres," IEEE J. Lightw. Technol., vol. 6, no. 1, pp. 17-20, Jan. 1988.

[20] D. Iannuzzi, S. Deladi, V. J. Gadgil, R. G. P. Sanders, H. Schreuders, and M. C. Elwenspoek, "Monolithic fiber-top sensor for critical environments and standard applications," Appl. Phys. Lett., vol. 88, no. 5, pp. 053501-1-053501-3, 2006.

[21] D. Chavan, G. Gruca, S. de Man, M. Slaman, J. H. Rector, K. Heeck, and D. Iannuzzi, "Ferrule-top atomic force microscope," Rev. Sci. Instrum., vol. 81 , no. 12 , pp. 123702-1-123702-5, 2010. 
[22] J.-L. Kou, J. Feng, Q.-J. Wang, F. Xu, and Y.-Q. Lu, "Microfiber-probebased ultrasmall interferometric sensor," Opt. Lett., vol. 35, no. 13, pp. 2308-2310, 2010.

[23] J.-L. Kou, S.-J. Qiu, F. Xu, Y.-Q. Lu, Y. Yuan, and G. Zhao, "Miniaturized metal-dielectric-hybrid tapered fiber tip grating for refractive index sensing," IEEE Photon. Technol. Lett., vol. 23, no. 22, pp. 1712-1714, Nov. 2011.

[24] J.-L. Kou, S.-J. Qiu, F. Xu, and Y.-Q. Lu, "Demonstration of a compact temperature sensor based on first-order Bragg grating in a tapered fiber probe," Opt. Exp., vol. 19, no. 19, pp. 18452-18457, 2011.

Jun-long Kou is currently pursuing the Masters degree with the College of Engineering and Applied Sciences and the National Laboratory of Solid State Microstructures, Nanjing University, Nanjing, China.

His current research interests include nonlinear optics and fiber-optics.

Wei Guo is currently pursuing the Masters degree with the College of Engineering and Applied Sciences and the National Laboratory of Solid State Microstructures, Nanjing University, Nanjing, China.

His current research interests include nonlinear optics and fiber-optics.
Fei Xu received the Ph.D. degree from the Optoelectronics Research Center, University of Southampton, Southampton, U.K., in 2008.

$\mathrm{He}$ is currently an Associate Professor with the College of Engineering and Applied Sciences and the National Laboratory of Solid State Microstructures, Nanjing University, Nanjing, China. He has published over 50 peer-reviewed papers on opto-electronic materials and devices. His current research interests include nano-photonics and fiber-optics.

Yan-qing Lu (SM'03) is currently a Professor with the College of Engineering and Applied Sciences and the National Laboratory of Solid State Microstructures, Nanjing University, Nanjing, China.

He holds 15 U.S. and Chinese patents. He has published over 80 peerreviewed papers on opto-electronic materials and devices. His current research interests include fiber optics, nano-photonics, and liquid crystal devices. 